\title{
ADIABATIC INVARIANTS FOR STRINGS AND MEMBRANES SUBJECTED TO SLOWLY-VARYING TENSION*
}

BY

W. A. DAY

Hertford College, Oxford

1. Introduction. In 1911, in the early days of the quantum theory, Lorentz was led to consider a certain problem of classical mechanics, viz. the motion of a pendulum whose length changes slowly. A mechanical clock, affected by seasonal variations in temperature, provides an illustration of what Lorentz had in mind.

The motion is described by the differential equation

$$
\ddot{x}+\omega(t)^{2} x=0,
$$

in which $\omega / 2 \pi$ is the local frequency of vibration. It was conjectured that the ratio

$$
I=\left(\omega^{2} x^{2}+\dot{x}^{2}\right) / \omega
$$

is approximately constant, or, in other words, that the energy is approximately proportional to the frequency - the changes in which may be large over large intervals of time. In short, it was conjectured that $I$ must be an adiabatic invariant for Lorentz's problem.

Heuristic justification for the conjecture was made to depend upon the identification of $I$ as an action variable of Hamiltonian mechanics.

Rigorous justification was provided later: it is sufficient to refer the reader to Littlewood's paper [1], which is reprinted in [2], together with that author's other papers on adiabatic invariance, and comments by Swinnerton-Dyer.

Swinnerton-Dyer [2, p. 426] remarks that Littlewood's methods are highly specific to Lorentz's problem. It turns out, though, that they rest upon certain identities, some of which have counterparts in, and applications to, a wider field.

An instance of application to a wider field is provided by the transverse vibration of a membrane, stretched to a tension $\tau(t)$ which is slowly-varying. In this case, the displacement $u(x, y, t)$ is a solution of the wave equation

$$
\tau\left(\frac{\partial^{2} u}{\partial x^{2}}+\frac{\partial^{2} u}{\partial y^{2}}\right)=\rho \frac{\partial^{2} u}{\partial t^{2}},
$$

$\rho(x, y)$ being the mass density.

* Received May 29, 1986.

(c) 1987 Brown University 
It will be shown that, provided the boundary of the membrane is fixed,

$$
\tau^{-1 / 2} \iint\left[\tau\left(\frac{\partial u}{\partial x}\right)^{2}+\tau\left(\frac{\partial u}{\partial y}\right)^{2}+\rho\left(\frac{\partial u}{\partial t}\right)^{2}\right] d x d y
$$

is an adiabatic invariant, i.e., the energy is approximately proportional to the square root of the tension.

It will be shown, in addition, that

$$
\tau^{-3 / 2} \iint\left[\tau\left(\frac{\partial^{2} u}{\partial x \partial t}\right)^{2}+\tau\left(\frac{\partial^{2} u}{\partial y \partial t}\right)^{2}+\rho\left(\frac{\partial^{2} u}{\partial t^{2}}\right)^{2}\right] d x d y
$$

is a further example of an adiabatic invariant; in this case the higher-order energy, obtained by replacing $u$ by its derivative with respect to $t$, is approximately proportional to the cube of the square root of the tension.

2. An adiabatic invariant for hyperbolic equations. The results of this section and the next have considerable generality - much more than is needed to cover applications to strings and membranes. In order not to introduce too cumbersome a system of notation, the discussion will be confined to a single hyperbolic equation in one unknown $u(x, t)=$ $u\left(x_{1}, \ldots, x_{n}, t\right)$; it will be apparent, though, that the methods extend to certain hyperbolic systems and, notably, to the equations of dynamic linear elasticity, if some mechanism is available which makes the elastic moduli slowly-varying functions of the time.

Let $D$ be a domain in $n$-dimensional space, i.e., a set that is bounded, open, and connected. Let $\bar{D}$ be its closure, and let its boundary $\partial D$ be smooth. Let $L$ be the differential operator

$$
\sum_{i=1}^{n} \sum_{j=1}^{n} \frac{\partial}{\partial x_{i}}\left(a_{i j}(x) \frac{\partial}{\partial x_{j}}\right)
$$

The operator is required to be formally self-adjoint and uniformly elliptic: thus $a_{i j}=a_{j i}$, and there is a positive constant $\lambda$ such that

$$
\sum_{i=1}^{n} \sum_{j=1}^{n} a_{i j}(x) \xi_{i} \xi_{j} \geqslant \lambda \sum_{i=1}^{n} \xi_{i}^{2}
$$

It will be supposed that $u(x, t)$ is defined whenever $x$ is a point of $\bar{D}$ and $0 \leqslant t<\infty$, that it has an appropriate number of continuous derivatives, and that it is a solution of the hyperbolic equation

$$
\phi L u=b \partial^{2} u / \partial t^{2},
$$

which also satisfies the boundary condition

$$
u(x, t)=0 \quad \text { if } x \text { is a point of } \partial D \text { and } 0 \leqslant t<\infty,
$$

and the initial conditions

$$
u(x, 0)=u_{0}(x), \frac{\partial u}{\partial t}(x, 0)=v_{0}(x) \quad \text { if } x \text { is a point of } \bar{D}
$$

Here $b(x)$ is taken to be positive on $\bar{D}$ and independent of $t$. 
The coefficient $\phi(t)$ is required to be positive, to start at the initial value $\phi(0)=1$, and to tend slowly, as $t \rightarrow \infty$, to a limiting value $\phi(\infty)$, which need not be close to the initial value. In precise terms, $\phi$ will be said to be slowly-varying if $\phi(0)=1$, if $\phi \rightarrow \phi(\infty)$, $\dot{\phi} \rightarrow 0, \ddot{\phi} \rightarrow 0$ as $t \rightarrow \infty$, and if there are positive constants $\varepsilon$ and $c_{k}(k=1, \ldots, 7)$ such that

$$
\begin{gathered}
c_{1} \leqslant \phi \leqslant c_{2}, \quad|\dot{\phi}| \leqslant c_{3} \varepsilon, \quad|\ddot{\phi}| \leqslant c_{4} \varepsilon^{2}, \\
\int_{0}^{\infty}|\dot{\phi}| d t \leqslant c_{5}, \quad \int_{0}^{\infty}|\ddot{\phi}| d t \leqslant c_{6} \varepsilon, \quad \int_{0}^{\infty}|\dddot{\phi}| d t \leqslant c_{7} \varepsilon^{2} .
\end{gathered}
$$

These conditions are satisfied, in particular, whenever $\phi(t)=\Phi(\varepsilon t)$, where $\Phi(0)=1$, $\Phi \rightarrow \Phi(\infty), \dot{\Phi} \rightarrow 0, \ddot{\Phi} \rightarrow 0$ as $t \rightarrow \infty$, and

$$
\begin{aligned}
& c_{1} \leqslant \Phi \leqslant c_{2}, \quad|\dot{\Phi}| \leqslant c_{3},|\ddot{\Phi}| \leqslant c_{4}, \\
& \int_{0}^{\infty}|\dot{\Phi}| d t \leqslant c_{5}, \quad \int_{0}^{\infty}|\ddot{\Phi}| d t \leqslant c_{6}, \quad \int_{0}^{\infty}|\ddot{\Phi}| d t \leqslant c_{7} .
\end{aligned}
$$

The constant $\varepsilon$ measures the slowness with which $\phi$ varies, and it will be helpful to think of the limiting value $\phi(\infty)$ and the constants $c_{k}$ as being fixed, while the positive parameter $\varepsilon$ tends to 0 . Any statement of the form

$$
f=O(g(\varepsilon))
$$

where $f(t)$ is defined on an interval $\delta \leqslant t<\infty$ ( $\delta=0$ in all but one of our applications), is to be interpreted as meaning that

$$
|f(t)| \leqslant B g(\varepsilon), \quad \delta \leqslant t<\infty,
$$

where $B$ is a positive constant that is independent of $\varepsilon$, but may depend upon the limit $\phi(\infty)$, the constants $c_{k}$, the domain $D$, the coefficients $a_{i j}$ and $b$, or the initial data $u_{0}$ and $v_{0}$.

It is easily checked that when $\phi$ is slowly-varying the same is true of $\psi=\phi^{p}$, where $p$ is any real exponent (the cases $p=-1 / 2$ and $p=-3 / 2$ are the ones of interest). The constants $d_{k}$ that appear in the inequalities

$$
\begin{array}{cc}
d_{1} \leqslant \psi \leqslant d_{2}, \quad|\dot{\psi}| \leqslant d_{3} \varepsilon, & |\ddot{\psi}| \leqslant d_{4} \varepsilon^{2}, \\
\int_{0}^{\infty}|\dot{\psi}| d t \leqslant d_{5}, \quad \int_{0}^{\infty}|\ddot{\psi}| d t \leqslant d_{6} \varepsilon, & \int_{0}^{\infty}|\ddot{\psi}| d t \leqslant d_{7} \varepsilon^{2},
\end{array}
$$

are determined by the $c_{k}$ and $p$, but $d_{k} \neq c_{k}$ in general.

Taking Littlewood's methods as a guide, let us introduce the integrals

$$
\begin{aligned}
& E=J+K \\
& F=\int_{D} b u^{2} d x \\
& J=\phi \int_{D} \sum_{i=1}^{n} \sum_{j=1}^{n} a_{i j} \frac{\partial u}{\partial x_{i}} \frac{\partial u}{\partial x_{j}} d x, \\
& K=\int_{D} b\left(\frac{\partial u}{\partial t}\right)^{2} d x .
\end{aligned}
$$


To within irrelevant factors, $J$ is the potential energy, $K$ is the kinetic energy, and $E$ is the total energy.

The key step in the construction of an adiabatic invariant is to recognize that the derivative of $E$ can be calculated by using either of the formulas

$$
\begin{gathered}
\dot{E}=(\dot{\phi} / \phi) J, \\
\dot{E}=(\dot{\phi} / \phi)\left(\frac{1}{2} E-\frac{1}{4} \ddot{F}\right) .
\end{gathered}
$$

The arguments needed to derive (2.1) and (2.2) are straightforward. Thus, if we multiply both sides of the hyperbolic equation by $2 \partial u / \partial t$, and rearrange the equation that results, we deduce that

$$
\begin{aligned}
2 \phi \sum_{i=1}^{n} \sum_{j=1}^{n} \frac{\partial}{\partial x_{i}}\left(\frac{\partial u}{\partial t} a_{i j} \frac{\partial u}{\partial x_{j}}\right)+\dot{\phi} \sum_{i=1}^{n} \sum_{j=1}^{n} a_{i j} & \frac{\partial u}{\partial x_{i}} \frac{\partial u}{\partial x_{j}} \\
& =\frac{\partial}{\partial t}\left(\phi \sum_{i=1}^{n} \sum_{j=1}^{n} a_{i j} \frac{\partial u}{\partial x_{i}} \frac{\partial u}{\partial x_{j}}+b\left(\frac{\partial u}{\partial t}\right)^{2}\right) .
\end{aligned}
$$

On integrating with respect to $x$, and using the fact that $u$ vanishes on the boundary, we then arrive at (2.1).

Next, observe that

$$
\begin{aligned}
\dot{F} & =2 \int_{D} b u \frac{\partial u}{\partial t} d x \\
\ddot{F} & =2 \int_{D} b\left(\frac{\partial u}{\partial t}\right)^{2} d x+2 \int_{D} b u \frac{\partial^{2} u}{\partial t^{2}} d x \\
& =2 K+2 \phi \int_{D} u \sum_{i=1}^{n} \sum_{j=1}^{n} \frac{\partial}{\partial x_{i}}\left(a_{i j} \frac{\partial u}{\partial x_{j}}\right) d x .
\end{aligned}
$$

If we use integration by parts, and the boundary condition, we see that

$$
\ddot{F}=2 K-2 J=2 E-4 J \text {. }
$$

Thus, we can replace $J$ by $\frac{1}{2} E-\frac{1}{4} \ddot{F}$ in (2.1), and when we do so we arrive at (2.2).

If (2.2) were to be regarded as a first-order differential equation for $E$, the appropriate integrating factor would be $\phi^{-1 / 2}$. Indeed, if we put $\psi=\phi^{-1 / 2}$ and

$$
I=\psi E,
$$

(2.2) reduces to the equation

$$
\dot{I}=\frac{1}{2} \dot{\psi} \ddot{F}
$$

which involves $I$ only through its derivative.

We shall continue to regard (2.1) as a formula for $\dot{E}$; expressed in terms of $\psi$, rather than $\phi$, it says that

$$
E=-(\dot{\psi} / 2 \psi) J
$$

It will be necessary to supplement (2.3), (2.4), and (2.5) with an estimate connecting $F$ and $J$. To derive it, we use the fact that $u$ vanishes on the boundary, and invoke Poincaré's inequality and the uniform ellipticity of $L$, to deduce the existence of a positive 
constant $\mu$ such that

$$
\int_{D} b u^{2} d x \leqslant \mu \int_{D} \sum_{i=1}^{n} \sum_{j=1}^{n} a_{i j} \frac{\partial u}{\partial x_{i}} \frac{\partial u}{\partial x_{j}} d x
$$

i.e.,

$$
F \leqslant \mu \psi^{2} J
$$

and this is the required estimate.

We are now in position to verify that $I$ is an adiabatic invariant by demonstrating that: the limit $I(\infty)$ exists, and

$$
I=I(\infty)+O(\varepsilon)
$$

In order to show that $I$ tends to a limit, it will be enough to show that $E$ tends to a limit $E(\infty)$, for (2.3) will then imply that $I \rightarrow I(\infty)=\psi(\infty) E(\infty)$.

Since $E=J+K \geqslant J,(2.5)$ tells us that

$$
|\dot{E}|=\frac{|\dot{\psi}|}{2 \psi} J \leqslant \frac{|\dot{\psi}|}{2 \psi} E \leqslant \frac{1}{2} d_{1}^{-1}|\dot{\psi}| E,
$$

and, therefore,

$$
|\dot{E}| \leqslant \frac{1}{2} d_{1}^{-1} d_{3} \varepsilon E \text {. }
$$

This last inequality ensures that $E$ is strictly positive everywhere on $0 \leqslant t<\infty$, or else vanishes identically. For, suppose it to be the case that $E\left(t_{0}\right)=0$ at some $t_{0}$. Let $t_{1}$ be any number greater than $t_{0}$, and let $M$ be the maximum value attained by $E$ on the interval $0 \leqslant t \leqslant t_{1}$. On this interval

$$
E(t)=\int_{t_{0}}^{t} \dot{E} d t \leqslant \frac{1}{2} d_{1}^{-1} d_{3} \varepsilon \int_{t_{0}}^{t} E d t,
$$

and an inductive argument establishes the estimates

$$
E(t) \leqslant \frac{M}{k !}\left[\frac{1}{2} d_{1}^{-1} d_{3} \varepsilon\left(t-t_{0}\right)\right]^{k}
$$

for every $k=1,2,3, \ldots$ On taking the limit as $k \rightarrow \infty$, we conclude that $E$ vanishes identically on every interval $0 \leqslant t \leqslant t_{1}$, with $t_{1}>t_{0}$; i.e., $E$ vanishes identically on $0 \leqslant t<\infty$ if it vanishes at some $t_{0}$.

If $E$ vanishes everywhere on $0 \leqslant t<\infty$, the same is true of $I$, and (2.7) is certainly correct in this case. Thus, $E$ may be supposed, henceforth, to be strictly positive.

Upon returning to $(2.8)$, we see that

$$
\left|\frac{\cdot}{\log E}\right| \leqslant \frac{1}{2} d_{1}^{-1}|\dot{\psi}|
$$

and, therefore, the integral

$$
\int_{0}^{\infty}\left|\frac{\cdot}{\log E}\right| d t
$$

converges, and does not exceed

$$
\frac{1}{2} d_{1}^{-1} \int_{0}^{\infty}|\dot{\psi}| d t \leqslant \frac{1}{2} d_{1}^{-1} d_{5} .
$$


The convergence of the integral guarantees that $\log E$ tends to a limit as $t \rightarrow \infty$ and, hence, $E$ itself and $I$ tend to limits.

The argument actually establishes a little more. Indeed,

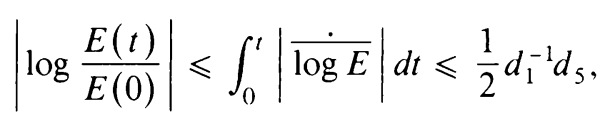

and, therefore,

$$
E(t) \leqslant E(0) \exp \left(\frac{1}{2} d_{1}^{-1} d_{5}\right),
$$

the right-hand side being independent of $\varepsilon$. If we recall our convention regarding the $O$-notation, we see that $E=O(1)$. It also follows that $F$ and $\dot{F}$ are $O(1)$. For, as the estimate (2.6) tells us,

$$
F \leqslant \mu \psi^{2} E \leqslant \mu d_{2}^{2} E=O(1)
$$

while the Schwarz inequality gives

$$
|\dot{F}|=2\left|\int_{D} b u \frac{\partial u}{\partial t} d x\right| \leqslant 2(F K)^{1 / 2} \leqslant 2(F E)^{1 / 2}=O(1) .
$$

To complete the proof, we let $T$ be any large positive number and return to (2.4). An integration by parts yields

$$
I(T)-I(t)=\frac{1}{2}[\dot{\psi} \dot{F}]_{t}^{T}-\frac{1}{2} \int_{t}^{T} \ddot{\psi} \dot{F} d t .
$$

Since $\dot{\psi}(T) \dot{F}(T) \rightarrow 0$ as $T \rightarrow \infty$, we have

$$
I(t)=I(\infty)+\frac{1}{2} \dot{\psi}(t) \dot{F}(t)+\frac{1}{2} \int_{t}^{\infty} \ddot{\psi} \dot{F} d t .
$$

Hence

$$
\begin{aligned}
|I(t)-I(\infty)| & \leqslant\left(\frac{1}{2}|\dot{\psi}(t)|+\frac{1}{2} \int_{t}^{\infty}|\ddot{\psi}| d t\right) O(1) \\
& \leqslant \frac{1}{2}\left(d_{3}+d_{6}\right)_{\varepsilon} O(1)=O(\varepsilon)
\end{aligned}
$$

which is (2.7).

3. Higher-order invariants. Just as the adiabatic invariant $I$ is constructed from the total energy $E$ by multiplying by a factor $\phi^{-1 / 2}$, so one might expect there to be a higher-order adiabatic invariant corresponding to each of the higher-order energies obtained by replacing $u$ by one of its derivatives with respect to $t$. This expectation is correct but, since higher-order invariants are likely to be of less physical interest than is $I$, the discussion will be restricted to only the simplest case, corresponding to a single differentiation with respect to $t$.

Let us put $v=\partial u / \partial t$. On differentiating the hyperbolic equation that $u$ satisfies, we obtain the hyperbolic equation

$$
\phi L v+\frac{\dot{\phi}}{\phi} b \frac{\partial v}{\partial t}=b \frac{\partial^{2} v}{\partial t^{2}}
$$


for $v$. Furthermore, $v$ satisfies the boundary condition

$$
v(x, t)=0 \quad \text { if } x \text { is a point of } \partial D \text { and } 0 \leqslant t<\infty,
$$

and the initial conditions

$$
v(x, 0)=v_{0}(x), \quad \frac{\partial v}{\partial t}(x, 0)=w_{0}(x), \quad \text { if } x \text { is a point of } \bar{D},
$$

where $w_{0}=b^{-1} L u_{0}$, as we see on setting $t=0$ in the equation that $u$ satisfies and remembering that $\phi(0)=1$.

Next we introduce the integrals

$$
\begin{aligned}
E_{1} & =J_{1}+K_{1} \\
F_{1} & =\int_{D} b v^{2} d x \\
J_{1} & =\phi \int_{D} \sum_{i=1}^{n} \sum_{j=1}^{n} a_{i j} \frac{\partial v}{\partial x_{i}} \frac{\partial v}{\partial x_{j}} d x \\
K_{1} & =\int_{D} b\left(\frac{\partial v}{\partial t}\right)^{2} d x .
\end{aligned}
$$

The second of these, $F_{1}$, coincides with the integral previously denoted by $K$.

The construction of an adiabatic invariant depends upon formulas for the derivative of $E_{1}$ which are akin to (2.1) and (2.2); these can be deduced by methods essentially the same as those already used, and prove to be

$$
\begin{aligned}
& \dot{E}_{1}=(\dot{\phi} / \phi)\left(J_{1}+2 K_{1}\right), \\
& \dot{E}_{1}=(\dot{\phi} / \phi)\left(\frac{3}{2} E_{1}+\frac{1}{4}\left(\ddot{F}_{1}-(\dot{\phi} / \phi) F_{1}\right)\right) .
\end{aligned}
$$

The integrating factor for (3.2) is $\psi=\phi^{-3 / 2}$, and $I_{1}=\phi^{-3 / 2} E_{1}$ is a candidate for being an adiabatic invariant. In fact: the limit $I_{1}(\infty)$ exists, and

$$
I_{1}=I_{1}(\infty)+O(\varepsilon)
$$

The proof proceeds on lines similar to that of Sec. 2 and involves using appropriate counterparts to (2.3), (2.4), (2.5), and (2.6); these are

$$
\begin{aligned}
& I_{1}=\psi E_{1}, \\
& \dot{I}_{1}=-\frac{1}{6} \dot{\psi} \ddot{F}_{1}-\frac{1}{9}\left(\dot{\psi}^{2} / \psi\right) \dot{F}_{1}, \\
& \dot{E}_{1}=-\frac{2}{3}(\dot{\psi} / \psi)\left(J_{1}+2 K_{1}\right), \\
& F_{1} \leqslant \mu \psi^{2 / 3} J_{1} .
\end{aligned}
$$

It follows from (3.6) that

$$
\left|\dot{E}_{1}\right|=\frac{2}{3} \frac{|\dot{\psi}|}{\psi}\left(J_{1}+2 K_{1}\right) \leqslant \frac{4}{3} \frac{|\dot{\psi}|}{\psi} E_{1} \leqslant \frac{4}{3} d_{1}^{-1}|\dot{\psi}| E_{2},
$$

and, therefore,

$$
\left|\dot{E}_{1}\right| \leqslant \frac{4}{3} d_{1}^{-1} d_{3} \varepsilon E_{1} \text {. }
$$

It is now possible to deduce, just as before, that the limits $E_{1}(\infty)$ and $I_{1}(\infty)$ exist, and that each of $E_{1}, F_{1}$, and $\dot{F}_{1}$ is $O(1)$. 
Now let $T$ be any large positive number. On integrating (3.5) by parts, we obtain the equation

$$
I_{1}(T)-I_{1}(t)=-\left[\frac{1}{6} \dot{\psi} \dot{F}_{1}+\frac{1}{9} \frac{\dot{\psi}^{2}}{\psi} F_{1}\right]_{t}^{T}+\int_{t}^{T}\left(\frac{1}{6} \ddot{\psi} \dot{F}_{1}+\frac{1}{9}\left(\frac{2 \dot{\psi} \ddot{\psi}}{\psi}-\frac{\dot{\psi}^{3}}{\psi^{2}}\right) F_{1}\right) d t,
$$

and in the limit as $T \rightarrow \infty$ this yields

$I_{1}(t)=I_{1}(\infty)-\frac{1}{6} \dot{\psi}(t) \dot{F}_{1}(t)-\frac{1}{9} \frac{\dot{\psi}(t)^{2}}{\psi(t)} F_{1}(t)-\int_{t}^{\infty}\left(\frac{1}{6} \ddot{\psi} \dot{F}_{1}+\frac{1}{9}\left(\frac{2 \dot{\psi} \ddot{\psi}}{\psi}-\frac{\dot{\psi}^{3}}{\psi^{2}}\right) F_{1}\right) d t$

The required order relation (3.3) follows in a straightforward way.

4. Higher-order equations. The methods of Secs. 2 and 3 extend to certain higher-order equations that are of interest. It will be enough to illustrate what happens when the dimension $n=1$, when the domain $D$ is the interval $0<x<1$, and when $u(x, t)$ is a solution of the fourth-order equation

$$
\phi L u+b \frac{\partial^{2} u}{\partial t^{2}}=0
$$

in which $L$ is the operator

$$
\frac{\partial^{2}}{\partial x^{2}}\left(a(x) \frac{\partial^{2}}{\partial x^{2}}\right)
$$

$a(x)$ and $b(x)$ are positive in $0 \leqslant x \leqslant 1$, and $\phi(t)$ is slowly-varying. The boundary conditions are taken to be

$$
u(0, t)=\frac{\partial u}{\partial x}(0, t)=u(1, t)=\frac{\partial u}{\partial x}(1, t)=0, \quad 0 \leqslant t<\infty,
$$

and the initial conditions to be

$$
u(x, 0)=u_{0}(x), \quad \frac{\partial u}{\partial t}(x, 0)=v_{0}(x), \quad 0 \leqslant x \leqslant 1 .
$$

Upon introducing the integrals

$$
\begin{aligned}
& E=J+K \\
& F=\int_{0}^{1} b u^{2} d x \\
& J=\phi \int_{0}^{1} a\left(\frac{\partial^{2} u}{\partial x^{2}}\right)^{2} d x \\
& K=\int_{0}^{1} b\left(\frac{\partial u}{\partial t}\right)^{2} d x
\end{aligned}
$$

we find the derivative of $E$ to be given by precisely the same formulas as before, viz. (2.1) and (2.2). Furthermore, the boundary conditions at $x=0$ ensure that

$$
u(x, t)=\int_{0}^{x}(x-y) \frac{\partial^{2} u}{\partial y^{2}}(y, t) d y
$$


and, hence, that there is a constant $\mu$ such that

$$
\int_{0}^{1} b u^{2} d x \leqslant \mu \int_{0}^{1} a\left(\frac{\partial^{2} u}{\partial x^{2}}\right)^{2} d x, \text { i.e., } \quad F \leqslant \mu \phi^{-1} J .
$$

The argument now proceeds exactly as in Sec. 2 and establishes that

$$
\phi^{-1 / 2} \int_{0}^{1}\left(\phi a\left(\frac{\partial^{2} u}{\partial x^{2}}\right)^{2}+b\left(\frac{\partial u}{\partial t}\right)^{2}\right) d x
$$

is an adiabatic invariant.

5. Improvement by averaging. According to (2.7), the difference $I-I(\infty)$ is $O(\varepsilon)$. The purpose of this final section is to show that, in special circumstances, the error can be reduced to $O\left(\varepsilon^{2}\right)$ by taking an average over a local period. Such an averaging procedure makes sense only if $u(x, t)$ is periodic in $t$ when $\phi$ is exactly constant but, by contrast with Lorentz's problem, for which $x(t)$ is periodic if $\omega$ is constant, this is not generally so. It is so, however, for the transverse vibration of a homogeneous string and, as we shall verify, the averaging procedure is effective in that case.

For ease of exposition, let the domain $D$ be the interval $0<x<1$, let the operator $L=\partial^{2} / \partial x^{2}$, and let the coefficient $b(x)=1$. The differential equation is now

$$
\phi \frac{\partial^{2} u}{\partial x^{2}}=\frac{\partial^{2} u}{\partial t^{2}}
$$

where $\phi(t)$ is slowly-varying, the boundary conditions are

$$
u(0, t)=u(1, t)=0, \quad 0 \leqslant t<\infty,
$$

and the initial conditions are

$$
u(x, 0)=u_{0}(x), \quad \frac{\partial u}{\partial t}(x, 0)=v_{0}(x), \quad 0 \leqslant x \leqslant 1 .
$$

The various integrals of Sec. 2 reduce to

$$
\begin{aligned}
& E=J+K \\
& F=\int_{0}^{1} u^{2} d x \\
& J=\phi \int_{0}^{1}\left(\frac{\partial u}{\partial x}\right)^{2} d x \\
& K=\int_{0}^{1}\left(\frac{\partial u}{\partial t}\right)^{2} d x
\end{aligned}
$$

and the adiabatic invariant is

$$
I=\phi^{-1 / 2} \int_{0}^{1}\left(\phi\left(\frac{\partial u}{\partial x}\right)^{2}+\left(\frac{\partial u}{\partial t}\right)^{2}\right) d x
$$

The boundary conditions imply that

$$
\int_{0}^{1} u^{2} d x \leqslant \frac{1}{\pi^{2}} \int_{0}^{1}\left(\frac{\partial u}{\partial x}\right)^{2} d x
$$

the constant $1 / \pi^{2}$ being the optimum value for $\mu$. 
The local period is $2 \phi^{-1 / 2}=2 \psi$, where $\psi$ is the integrating factor associated with $I$. The improvement which results from averaging will be shown to be

$$
\frac{1}{2 \psi(t)} \int_{t-\psi(t)}^{t+\psi(t)} I(s) d s=I(\infty)+O\left(\varepsilon^{2}\right)
$$

It should be noted that the average is not defined for all $t$ in $0 \leqslant t<\infty$ but only for those with $t \geqslant \psi(t)$; it is certainly defined on the interval $d_{2} \leqslant t<\infty$, however.

The first step in the proof of (5.1) is to show that $u(x, t)$ comes close to being periodic in $t$. Let $x_{0}$ and $t_{0}$ be numbers such that $0<x_{0}<1$ and $t_{0} \geqslant \psi\left(t_{0}\right)$ but are otherwise arbitrary. Let $P^{+}$be the parallelogram in the $x, t$-plane whose vertices are $\left(1-x_{0}, t_{0}\right)$, $\left(1, t_{0}+\psi\left(t_{0}\right) x_{0}\right),\left(x_{0}, t_{0}+\psi\left(t_{0}\right)\right),\left(0, t_{0}+\psi\left(t_{0}\right)\left(1-x_{0}\right)\right)$, and let $P^{-}$be the parallelogram whose vertices are $\left(1-x_{0}, t_{0}\right),\left(0, t_{0}-\psi\left(t_{0}\right)\left(1-x_{0}\right)\right),\left(x_{0}, t_{0}-\psi\left(t_{0}\right)\right),\left(1, t_{0}-\right.$ $\left.\psi\left(t_{0}\right) x_{0}\right)$. The edges of both parallelograms are lines on which $t+\psi\left(t_{0}\right) x$ is constant, or $t-\psi\left(t_{0}\right) x$ is constant; the parallelograms have exactly one point in common, viz. the vertex $\left(1-x_{0}, t_{0}\right)$, and each has a vertex on the line $x=0$ and a vertex on the line $x=1$.

According to Green's theorem,

$$
\begin{aligned}
& \int_{P^{+}}\left(\frac{\partial u}{\partial t} d x+\psi\left(t_{0}\right)^{-2} \frac{\partial u}{\partial x} d x\right)=\iint_{P}\left(\psi\left(t_{0}\right)^{-2} \frac{\partial^{2} u}{\partial x^{2}}-\frac{\partial^{2} u}{\partial t^{2}}\right) d x d t \\
& \int_{P}\left(\frac{\partial u}{\partial t} d x+\psi\left(t_{0}\right)^{-2} \frac{\partial u}{\partial x} d t\right)=\iint_{P}\left(\psi\left(t_{0}\right)^{-2} \frac{\partial^{2} u}{\partial x^{2}}-\frac{\partial^{2} u}{\partial t^{2}}\right) d x d t
\end{aligned}
$$

On evaluating the line integrals, and remembering that $u$ vanishes on the lines $x=0$ and $x=1$, we deduce the equations

$$
\begin{aligned}
& u\left(x_{0}, t_{0}+\psi\left(t_{0}\right)\right)+u\left(1-x_{0}, t_{0}\right)=\frac{1}{2} \psi\left(t_{0}\right) \iint_{P^{+}}\left(\frac{\partial^{2} u}{\partial t^{2}}-\psi\left(t_{0}\right)^{-2} \frac{\partial^{2} u}{\partial x^{2}}\right) d x d t, \\
& u\left(x_{0}, t_{0}-\psi\left(t_{0}\right)\right)+u\left(1-x_{0}, t_{0}\right)=\frac{1}{2} \psi\left(t_{0}\right) \iint_{P^{-}}\left(\frac{\partial^{2} u}{\partial t^{2}}-\psi\left(t_{0}\right)^{-2} \frac{\partial^{2} u}{\partial x^{2}}\right) d x d t .
\end{aligned}
$$

When we subtract the second from the first, the contributions from the common vertex cancel, and we are left with the formula

$$
\begin{aligned}
& u\left(x_{0}, t_{0}+\psi\left(t_{0}\right)\right)-u\left(x_{0}, t_{0}-\psi\left(t_{0}\right)\right) \\
& =\frac{1}{2} \psi\left(t_{0}\right)\left(\iint_{P^{+}}-\iint_{P}\right)\left(\frac{\partial^{2} u}{\partial t^{2}}-\psi\left(t_{0}\right)^{-2} \frac{\partial^{2} u}{\partial x^{2}}\right) d x d t .
\end{aligned}
$$

The differential equation permits the substitution of $\psi^{2} \partial^{2} u / \partial t^{2}$ for $\partial^{2} u / \partial x^{2}$, and so

$$
\begin{aligned}
u\left(x_{0}, t_{0}+\psi\left(t_{0}\right)\right)-u\left(x_{0}, t_{0}-\psi\left(t_{0}\right)\right) & \\
= & \frac{1}{2 \psi\left(t_{0}\right)}\left(\iint_{P^{+}}-\iint_{P^{-}}\right)\left(\psi\left(t_{0}\right)^{2}-\psi(t)^{2}\right) \frac{\partial^{2} u}{\partial t^{2}} d x d t .
\end{aligned}
$$

If $\phi$, and hence $\psi$, were constant, (5.2) would imply exact periodicity of $u$. 
It is proposed to use (5.2) to show that

$$
F(t+\psi(t))-F(t-\psi(t))=O(\varepsilon) .
$$

Because $P^{+}$and $P^{-}$lie within the rectangle $0 \leqslant x \leqslant 1,\left|t-t_{0}\right| \leqslant \psi\left(t_{0}\right)$, the absolute value of the right-hand side of (5.2) does not exceed

$$
\frac{1}{2 \psi\left(t_{0}\right)} \int_{t_{0}-\psi\left(t_{0}\right)}^{t_{0}+\psi\left(t_{0}\right)} \int_{0}^{1}\left|\psi(t)^{2}-\psi\left(t_{0}\right)^{2}\right|\left|\frac{\partial^{2} u}{\partial t^{2}}\right| d x d t
$$

On introducing the higher-order kinetic energy

$$
K_{1}=\int_{0}^{1}\left(\frac{\partial^{2} u}{\partial t^{2}}\right)^{2} d x
$$

using the Schwarz inequality, and noting that if $\left|t-t_{0}\right| \leqslant \psi\left(t_{0}\right)$,

$$
\begin{aligned}
\frac{1}{2} \psi\left(t_{0}\right)^{-1}\left|\psi(t)^{2}-\psi\left(t_{0}\right)^{2}\right| & =\psi\left(t_{0}\right)^{-1}\left|\int_{t_{0}}^{t} \psi \dot{\psi} d t\right| \\
& \leqslant \psi\left(t_{0}\right)^{-1} \int_{t_{0}}^{t_{0}+\psi\left(t_{0}\right)} d_{2} d_{3} \varepsilon d t=d_{2} d_{3} \varepsilon
\end{aligned}
$$

we deduce that

$$
\left|u\left(x_{0}, t_{0}+\psi\left(t_{0}\right)\right)-u\left(x_{0}, t_{0}-\psi\left(t_{0}\right)\right)\right| \leqslant d_{2} d_{3} \varepsilon \int_{t_{0}-\psi\left(t_{0}\right)}^{t_{0}+\psi\left(t_{0}\right)} K_{1}^{1 / 2} d t,
$$

or, on replacing $x_{0}$ and $t_{0}$ by general $x$ and $t$, that

$$
|u(x, t+\psi(t))-u(x, t-\psi(t))| \leqslant d_{2} d_{3} \varepsilon \int_{t-\psi(t)}^{t+\psi(t)} K_{1}^{1 / 2} d t
$$

Squaring both sides of this last inequality, integrating with respect to $x$, and using the Schwarz inequality again yields the estimate

$$
\int_{0}^{1}|u(x, t+\psi(t))-u(x, t-\psi(t))|^{2} d x \leqslant 2\left(d_{2} d_{3} \varepsilon\right)^{2} \psi(t) \int_{t-\psi(t)}^{t+\psi(t)} K_{1} d t .
$$

The arguments of Sec. 3 show that the higher-order energy $E_{1}$ is $O(1)$, and the same must be true of $K_{1}\left(\leqslant E_{1}\right)$. This fact and the bound $\psi \leqslant d_{2}$ show that the right-hand side of (5.4) is $O\left(\varepsilon^{2}\right)$. On the other hand, if we take the square root of each side of (5.4), and then estimate the left-hand side from below with the help of the triangle inequality $\|f-g\| \geqslant|\|f\|-\|g\||$, where $\|f\|$ is the $L^{2}$-norm $\left(\int_{0}^{1} f^{2} d x\right)^{1 / 2}$, we conclude that

$$
F(t+\psi(t))^{1 / 2}-F(t-\psi(t))^{1 / 2}=O(\varepsilon) .
$$

Since

$$
\begin{aligned}
F(t+\psi(t))-F(t-\psi(t))=\left(F(t+\psi(t))^{1 / 2}+\right. & \left.F(t-\psi(t))^{1 / 2}\right) \\
& \cdot\left(F(t+\psi(t))^{1 / 2}-F(t-\psi(t))^{1 / 2}\right),
\end{aligned}
$$

and $F$ is known to be $O(1)$, the order relation (5.3) is correct. 
The final step in the proof of (5.1) involves returning to (2.10), which, by a further integration by parts, gives

$$
\begin{aligned}
I(t) & =I(\infty)+\frac{1}{2} \dot{\psi}(t) \dot{F}(t)-\frac{1}{2} \ddot{\psi}(t) F(t)-\frac{1}{2} \int_{t}^{\infty} \dddot{\psi} F d t \\
& =I(\infty)+\frac{1}{2} \dot{\psi}(t) \dot{F}(t)+O\left(\varepsilon^{2}\right) .
\end{aligned}
$$

On averaging both sides, we find

$$
\begin{aligned}
\frac{1}{2 \psi(t)} \int_{t-\psi(t)}^{t+\psi(t)} I(s) d s= & I(\infty)+\frac{1}{4 \psi(t)} \int_{t-\psi(t)}^{t+\psi(t)} \dot{\psi}(s) \dot{F}(s) d s+O\left(\varepsilon^{2}\right) \\
= & I(\infty)+\frac{1}{4 \psi(t)} \int_{t-\psi(t)}^{t+\psi(t)}(\dot{\psi}(s)-\dot{\psi}(t)) \dot{F}(s) d s \\
& +\frac{1}{4} \psi(t)^{-1} \dot{\psi}(t)(F(t+\psi(t))-F(t-\psi(t)))+O\left(\varepsilon^{2}\right) \\
= & I(\infty)+\frac{1}{4 \psi(t)} \int_{t-\psi(t)}^{t+\psi(t)} \dot{F}(s)\left[\int_{t}^{s} \ddot{\psi}\left(s^{\prime}\right) d s^{\prime}\right] d s \\
& +\frac{1}{4} \psi(t)^{-1} \dot{\psi}(t)(F(t+\psi(t))-F(t-\psi(t)))+O\left(\varepsilon^{2}\right) .
\end{aligned}
$$

With the help of (5.3), the third term in this sum is easily seen to be $O\left(\varepsilon^{2}\right)$; the same is true of the second term and, hence, the proof is complete.

\section{REFERENCES}

[1] J. E. Littlewood, Lorentz's pendulum problem, Ann. Physics 21, 233-242 (1963)

[2] Collected papers of J. E. Littlewood, vol. I, edited by a committee appointed by the London Mathematical Society, Clarendon Press, Oxford, 1982 